Antimatter and Gravity (WAG 2013)

International Journal of Modern Physics: Conference Series

Vol. 30 (2014) 1460254 (9 pages)

(C) The Author

DOI: $10.1142 / \mathrm{S} 2010194514602543$

\title{
Testing the weak equivalence principle with macroscopic proof masses on ground and in space: A brief review
}

\author{
Anna M. Nobili \\ Department of Physics "E. Fermi", University of Pisa and INFN \\ Largo Bruno Pontecorvo 3, 56127 Pisa, Italy \\ nobili@dm.unipi.it
}

Received 10 February 2014

Revised 10 March 2014

Published 7 May 2014

\begin{abstract}
General relativity is founded on the experimental fact that in a gravitational field all bodies fall with the same acceleration regardless of their mass and composition. This is the weak equivalence principle, or universality of free fall. Experimental evidence of a violation would require either that general relativity is to be amended or that another force of nature is at play. In 1916 Einstein brought as evidence the torsion balance experiments by Eötvös, to $10^{-8}-10^{-9}$. In the 1960 s and early 70 s, by exploiting the "passive" daily rotation of the Earth, torsion balance tests improved to $10^{-11}$ and $10^{-12}$. More recently, active rotation of the balance at higher frequencies has reached $10^{-13}$. No other experimental tests of general relativity are both so crucial for the theory and so precise and accurate. If a similar differential experiment is performed inside a spacecraft passively stabilized by $1 \mathrm{~Hz}$ rotation while orbiting the Earth at $\simeq 600 \mathrm{~km}$ altitude the test would improve by 4 orders of magnitude, to $10^{-17}$, thus probing a totally unexplored field of physics. This is unique to weakly coupled concentric macroscopic test cylinders inside a rapidly rotating spacecraft.
\end{abstract}

Keywords: Tests of general relativity; equivalence principle; universality of free fall.

PACS Numbers: 04.80.Cc, 07.87.+v, 62.40.+i

\section{What is Special About the Universality of Free Fall and the Weak Equivalence Principle (UFF/WEP)?}

In 1687, in the opening paragraph of the Principia Newton stated the equivalence of inertial and gravitational mass on the basis of "... experiments on pendulums, very accurately made...". Within his theory the equations of motion of a body in

This is an Open Access article published by World Scientific Publishing Company. It is distributed under the terms of the Creative Commons Attribution 3.0 (CC-BY) License. Further distribution of this work is permitted, provided the original work is properly cited. 
the gravitational field of the Earth read:

$$
m_{i} \ddot{\vec{r}}=-\frac{G M m_{g}}{r^{3}} \vec{r}
$$

with $m_{i}, m_{g}$ the inertial and gravitational mass of the body and $M$ the gravitational mass of the Earth. If $m_{i}=m_{g}$ the equations require that all bodies in the field of the Earth acquire the same acceleration regardless of their mass and composition (universality of free fall - UFF). About 80 years earlier Galileo had tested UFF with pendulums of different composition. Newton made his own pendulum tests and concluded that inertial and gravitational mass must be equivalent (Newton's equivalence principle, later referred to as the weak equivalence principle - WEP). It is very likely that Galileo's and Newton's pendulums were measured to be subjected to the same acceleration to no better than 1 part in $10^{3}$. Yet, Newton's highly successful theory of gravity is founded on these experimental results.

In 1907 Einstein realized that all the natural laws except the law of gravity could be discussed within the framework of the theory of relativity he had published in 1905 (later to become the special theory of relativity). He then started his endeavor to include gravity, and later reported "the happiest thought in my life", which led to the formulation of the general theory of relativity:

"Just as is the case with the electric field produced by electromagnetic induction, the gravitational field has similarly only a relative existence. For if one considers an observer in free fall, e.g. from the roof of a house, there exists for him during his fall no gravitational field - at least in his immediate vicinity."

"... The breakthrough came suddenly one day. I was sitting on a chair in my patent office in Bern. Suddenly a thought struck me: If a man falls freely, he would not feel his weight. I was taken aback. This simple thought experiment made a big impression on me. This led me to the theory of gravity. ... I continued my thought: A falling man is accelerated. Then what he feels and judges is happening in the accelerated frame of reference. I decided to extend the theory of relativity to the reference frame with acceleration. I felt that in so doing I could solve the problem of gravity at the same time.

... It took me eight more years until I finally obtained the complete solution." (See Fig. 1 and Ref. 1 for details.)

In the 1916 paper $^{2}$ "The foundation of the general theory of relativity" Einstein showed how the physical equivalence between an accelerated frame and a uniform gravitational field, of which he became aware with his "happiest thought", leads to the general theory of relativity. In Sec. 2 of the paper he made it clear that this is "... made possible for us by the teaching of experience as to the existence of a field of force, namely the gravitational field, which possesses the remarkable property of imparting the same acceleration to all bodies."

It is apparent that this fact of nature must be proved experimentally. In the same years in which Einstein was developing the general theory of relativity, Eötvös for the first time ever used a torsion balance to test the universality of free fall. 

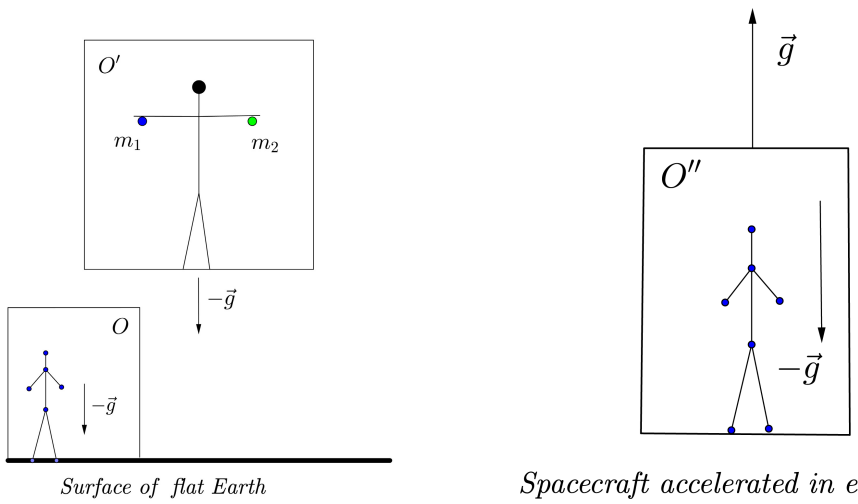

Spacecraft accelerated in empty space

Fig. 1. Observer $O$ sits on the surface of a flat non-rotating Earth; he is subjected to a uniform gravitational acceleration $-\vec{g}$ which, according to Newton's equivalence principle, is the same on all bodies regardless of their mass and composition. Observer $O^{\prime}$ sits inside a non-rotating elevator which is freely falling on a flat Earth which causes the same acceleration on all bodies; this gravitational field has no dynamical effects inside the lab and he does not feel his weight. Observer $O^{\prime \prime}$ sits inside a non-rotating spacecraft moving in empty space with constant acceleration $+\vec{g}$; the spacecraft is a non inertial frame and therefore all bodies inside it are subjected to an acceleration equal and opposite to that of the lab, i.e. $-\vec{g}$. In summary, as long as UFF holds i) labs $O$ and $O^{\prime \prime}$ are physically equivalent ii) inside lab $O^{\prime}$ gravity is eliminated.

The improvement over the best pendulum tests of the time was amazing, by several orders of magnitude. Einstein referred to Eötvös' experiments in a specific footnote: ${ }^{2}$ "Eötvös has proved experimentally that the gravitational field has this property in great accuracy", thus reminding the reader that the general theory of relativity relies on the experimental evidence of the universality of free fall.

\section{Why are WEP/UFF Tests so Sensitive?}

The physical quantity to be measured in UFF/WEP experiments is the relative acceleration $\Delta a=a_{1}-a_{2}$ of the free falling test masses relative to the source body. It's ratio to the average free fall acceleration of the two masses $a=\left(a_{1}+a_{2}\right) / 2-$ known as "the driving signal" — gives the dimensionless "Eötvös" parameter $\eta$ :

$$
\eta \equiv \frac{\Delta a}{a}
$$

that quantifies a deviation from UFF/WEP. If UFF/WEP holds, then $\Delta a=0$ and $\eta=0$; for a given value of the driving signal $a$, the smaller the measured differential acceleration $\Delta a$, the smaller the value of $\eta$, the more sensitive the test.

Since the signal being sought is a differential acceleration whose value must be zero (null experiment) there is in principle no need to measure the individual accelerations of the test masses. An experimental apparatus sensitive to the differential acceleration avoids the difficulties of deriving a very small number $(\Delta a)$ from the difference of two numbers $\left(a_{1}, a_{2}\right)$ which are many orders of magnitude larger. 
No absolute measurement is required when testing UFF/WEP. By comparison, when a very small gravitational acceleration is measured in order to establish the value of the gravitational constant $G$, in addition to making a very low noise experiment, capable of detecting a very small acceleration, the measured quantity must be compared with its theoretical prediction in order to establish the value of $G$. This requires knowledge and/or measurement of all the physical parameters involved in the model, which is much harder (see Ref. 3). The measurement of the gravitational redshift is another example of an absolute measurement (see Ref. 1 for a discussion on the universality of free fall, the equivalence principle, the gravitational redshift and the superior probing power of UFF/WEP tests).

\section{Why has the Torsion Balance Defeated Mass Dropping Tests?}

The best UFF/WEP tests so far, both in the field of the Earth and in the field of the Sun, have been performed in ground based laboratories using torsion balances (see Refs. 4-8).

The torsion balance is extremely sensitive to torques which twist the suspension fiber, hence to differential forces acting on test masses of different composition placed on opposite sides of the balance (the thinner the wire, the larger the twist angle, the more sensitive the balance).

It is also by design insensitive to all forces which are parallel to each other, of equal as well as of different size. This simple fact of physics holds at $1-g$. Consider a simple dumbbell balance with a thin fiber and two test bodies of different composition $A$ and $B$ at its ends. Any tiny torque directed along the wire will twist it. The total force $\vec{F}_{A}+\vec{F}_{B}$ acting on the balance, applied on its center of mass, is counterbalanced by the suspension wire which aligns itself with the direction of local gravity. Consider the total torque $\vec{M}=\vec{r}_{A} \times \vec{F}_{A}+\vec{r}_{B} \times \vec{F}_{B}\left(\vec{r}_{A}, \vec{r}_{B}\right.$ the position vectors of the test bodies separated by the relative position vector $\vec{r}=\vec{r}_{A}-\vec{r}_{B}$ along the dumbbell). The balance is sensitive only to the component of this torque along the direction of the wire, which is the direction of the total force $\vec{F}_{A}+\vec{F}_{B}$. This component is:

$$
M_{w}=\frac{\vec{r} \cdot \vec{F}_{A} \times \vec{F}_{B}}{\left|\vec{F}_{A}+\vec{F}_{B}\right|}
$$

hence, forces on the test bodies which are parallel to each other don't twist the fiber, which gets rid of an entire class of differential disturbances. Instead, if the ratio of inertial-to-gravitational mass is not the same for the suspended bodies (WEP/UFF violation) there will be residual forces on the bodies not parallel to each other which do twist the wire and therefore make the balance sensitive to a violation signal. However, "classical" non parallel gravitational forces remain which twist the fiber even in absence of violation, because the gravitational field is not uniform and test bodies on a balance are not concentric by design. Most of the care in torsion balance tests is concentrated in reducing these effects. 


\begin{tabular}{|c|c|c|c|c|c|}
\hline & Authors & Apparatus & Source mass & Materials & $\eta \equiv \Delta a / a$ \\
\hline \multirow[b]{3}{*}{$36 \mathrm{yr}$} & $\begin{array}{l}\text { Eötvös et al. } \approx 1900 \\
\text { collected in Ann. } \\
\text { Phys. } 1922\end{array}$ & $\begin{array}{l}\text { Torsion balance. Not } \\
\text { rotating. No signal } \\
\text { modulation }\end{array}$ & Earth & $\begin{array}{l}\text { Many } \\
\text { combinations }\end{array}$ & $10^{-8} \div 10^{-9}$ \\
\hline & $\begin{array}{l}\text { Roll, Krotkov \& Dicke } \\
\text { Ann. Phys. } 1964\end{array}$ & $\begin{array}{l}\text { Torsion balance. Not } \\
\text { rotating. } 24 \mathrm{hr} \\
\text { modulation by Earth } \\
\text { rotation }\end{array}$ & Sun & $\mathrm{Al}-\mathrm{Au}$ & $(1.3 \pm 1) \times 10^{-11}$ \\
\hline & $\begin{array}{l}\text { Braginsky \& Panov } \\
\text { JETP } 1972\end{array}$ & $\begin{array}{l}\text { Torsion balance. } \\
\text { 8TMs. Not rotating. } \\
\text { 24hr modulation by } \\
\text { Earth rotation }\end{array}$ & Sun & $\mathrm{Al}-\mathrm{Pt}$ & $(-0.3 \pm 0.9) \times 10^{-12}$ \\
\hline \multirow{4}{*}{$14 \mathrm{yr}$} & \multicolumn{5}{|c|}{ E. Fischbach et al.: "Reanalysis of the Eötvös Experiment" PRL 1986} \\
\hline & \multirow[t]{2}{*}{ Eöt-Wash, PRD 1994} & \multirow{2}{*}{$\begin{array}{l}\text { Rotating torsion } \\
\text { balance. } \approx 1 \mathrm{hr} \\
\text { modulation }\end{array}$} & \multirow{2}{*}{ Earth } & $\mathrm{Be}-\mathrm{Cu}$ & $(-1.9 \pm 2.5) \times 10^{-12}$ \\
\hline & & & & $\mathrm{Be}-\mathrm{Al}$ & $(-0.2 \pm 2.8) \times 10^{-12}$ \\
\hline & Eöt-Wash, PRL 1999 & $\begin{array}{l}\text { Rotating torsion } \\
\text { balance. } 1 \mathrm{hr} \text { to } 36 \\
\text { modulation }\end{array}$ & Sun & $\begin{array}{l}\text { Earthlike/ } \\
\text { Moonlike }\end{array}$ & $\begin{array}{l}\approx 10^{-12} \\
\left(\text { SEP } 1.3 \times 10^{-3}\right)\end{array}$ \\
\hline$\downarrow$ & Eöt-Wash, PRL 2008 & $\begin{array}{l}\text { Rotating torsion } \\
\text { balance. } \\
\text { 20' modulation }\end{array}$ & Earth & $\mathrm{Be}-\mathrm{Ti}$ & $(0.3 \pm 1.8) \times 10^{-13}$ \\
\hline
\end{tabular}

Fig. 2. (Color online) Improvements over the years of UFF/WEP tests performed with torsion balances. The best results have been obtained by rotating the torsion balance in order to up convert the target signal to higher frequency where noise is known to be lower.

Figire 2 shows how torsion balance tests of UFF/WEP have improved since the pioneer work of Eötvös in the early 1900. Since then, a major improvement by almost 3 orders of magnitude occurred in the 1960s when Dicke, ${ }^{4}$ soon followed by Braginsky, ${ }^{5}$ used a torsion balance to search for a violation in the field of the Sun, hence exploiting the "passive" daily rotation of the Earth to get a signal at $1.16 \cdot 10^{-5} \mathrm{~Hz}$ rather than DC. Next improvement came by actively rotating the torsion balance itself at a frequency faster than the rotation frequency of the Earth. This rotation up-converted the signal from both the Sun and the Earth to higher frequency and improved by another order of magnitude (see Refs. 6-8).

Figure 3 shows that in the field of the Earth the driving signal for mass dropping tests is about a factor 600 times stronger than it is for torsion balances. According to Eq. (2), this should result — for the same sensitivity to differential accelerations in a better UFF/WEP test by the same factor. Instead, mass dropping tests ${ }^{9}$ have reached $\Delta g / g=7.2 \cdot 10^{-10}$, which is not competitive with torsion balances by far, as shown in Fig. 2. Why is it so?

In most mass dropping experiments the test bodies are released individually and the result is affected by release errors. If the centers of mass at start are separated by a non zero distance along the direction to the source body the test masses free fall accelerations differ by a (classical) tidal acceleration which mimics a violation. The experiment ${ }^{9}$ was a very interesting attempt at making a mass dropping test differential. A vertical disk made of two halves of different material $(\mathrm{Al}$ and $\mathrm{Cu})$ was dropped inside an $8 \mathrm{~m}$ tall vacuum chamber at CERN and the rotation angle of the disk around its symmetry axis was read with low noise laser interferometry. 


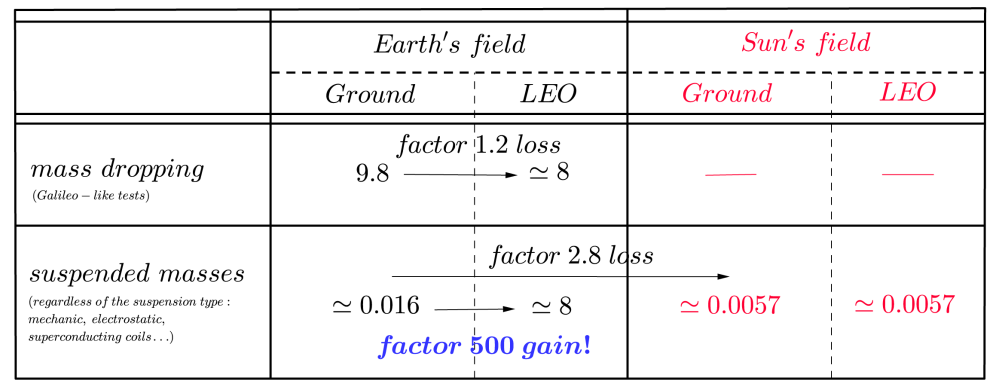

Fig. 3. (Color online) Strength of driving signal (in $\mathrm{ms}^{-2}$ ) for UFF/WEP experimental tests on ground and in low Earth orbit (LEO) at about $600 \mathrm{~km}$ altitude. The driving signal is the average free-fall acceleration of the test masses in the gravitational field of the source body. For experiments performed with suspended test masses the source body can be the Earth as well as the Sun.

If $\mathrm{UFF} / \mathrm{WEP}$ is violated the two half disks fall with different accelerations, resulting in a non zero rotation angle of the disk around its symmetry axis. Drops were performed also with a single material disk $(\mathrm{Al})$ and it was proved that the experiment was limited by the rotation imparted to the disk at release.

Another differential mass dropping experiment has been proposed ${ }^{10}$ to be performed inside a capsule released from a stratospheric balloon. In this case different composition test masses are mechanically coupled, their centers of mass are concentric, the pick up of their differential motion is capacitive and the sensor rotates in order to modulate the signal at the rotation frequency. The authors expect - at low temperature and thanks to $30 \mathrm{~s}$ of free fall time made possible by the balloon to be able to test UFF/WEP to $5 \cdot 10^{-15}$.

More recently a mass dropping test with independent initially concentric test masses, based on a laser interferometer gauge developed at Harvard-Smithsonian Center for Astrophysics, has been proposed to be performed in a sounding rocket. ${ }^{11}$ It envisages 8 drops of $120 \mathrm{~s}$ each with inversion of the payload and a careful monitoring of the test masses at release. The goal is a UFF/WEP to a few parts in $10^{17}$. A similar apparatus could be tested on ground over a shorter free fall time to assess the expected sensitivity.

\section{What can Space Offer and to Which Experiments?}

If a torsion balance experiment is performed inside a spacecraft orbiting the Earth at low altitude the driving signal of a UFF/WEP violation is a factor 500 stronger than on ground (see Fig. 3). This is by itself a factor 500 improvement in the "Eötvös" parameter (2) which quantifies the sensitivity of the test. Note that, as shown in Fig. 3, such advantage does not hold for Galileo mass dropping tests.

In addition, an instrument with weakly coupled test masses designed to detect differential accelerations (a "differential accelerometer") can be more sensitive in space than on ground provided that it has been designed to take full advantage of 
the peculiarities of the space environment. The most relevant ones are discussed below.

Obviously, in absence of weight test masses can be suspended and weakly coupled much more easily than at $1-g$, which makes them sensitive to very tiny differential accelerations. Suspending a $100 \mathrm{~kg}$ mass inside a small spacecraft in low earth orbit requires the same stiffness which on ground would sustain no more than 1 milligram!

However, a way of coupling the test masses different from the torsion balance must be devised. The torsion balance is sensitive to torques along the suspension wire, but at $0-g$ the total force is no longer aligned with the wire and Eq. (3) no longer holds.

Torsion balance UFF/WEP experiments on ground have shown that rotation (the faster the better) is crucial to perform a good test. Dicke and Braginsky have proved that "passive" rotation of the balance (passive because it rotates together with the lab and the whole Earth with one day period, needing no motor and no bearings) yields a leap in sensitivity despite the fact that the rotation frequency is very low and the driving signal from the Sun is even weaker than from the Earth. In space "passive" rotation is possible because the whole "laboratory" (the spacecraft) can spin together with the test masses by conservation of the initial angular momentum. For any rotating apparatus on ground this is obviously impossible; there will always be a rotating and a non rotating part, hence motor/bearings noise.

A spacecraft can be passively stabilized by rotation around its axis of maximum moment of inertia at a typical frequency of $1 \mathrm{~Hz}$, a factor 86400 faster than the Earth's rotation in Dicke's and Braginsky's experiments. If the rotation frequency of the spacecraft with respect to Earth is made to be the frequency of the target $\mathrm{UFF} / \mathrm{WEP}$ violation signal, this fact has far reaching consequences (see Sec. 5).

\section{5. "Galileo Galilei" (GG): A Differential Accelerometer inside a Spin-Axis Stabilized Spacecaft to Test UFF/WEP to $10^{-17}$}

$\mathrm{GG}^{12}$ has been designed following the drivers of Sec. 4. We need a balance with weakly coupled test masses sensitive to differential accelerations. Each test mass orbiting the Earth (the source body) is a 2-body gravitational problem with 2 degrees of freedom (in the orbit plane). Classical tidal (differential) accelerations between the centers of mass of the test bodies are minimized if they are concentric. The GG test masses are concentric co-axial cylinders very weakly coupled in the plane perpendicular to the symmetry/spin axis to form a $2 \mathrm{D}$ mechanical oscillator in rapid rotation. The test cylinders are hosted, in a nested configuration, inside a co-axial intermediate stage which in its turn is hosted by the spacecraft outer shell, all with cylindrical symmetry and spinning together — after initial spin up — by conservation of the angular momentum. No motor and no bearings are needed.

Weak coupling ensures high sensitivity to differential accelerations; fast rotation up-converts the signal from the orbital frequency $\left(1.7 \cdot 10^{-4} \mathrm{~Hz}\right)$ to the much higher 
spin frequency $(1 \mathrm{~Hz})$ where not only $1 / f$ electronics noise but also thermal noise from internal damping is much lower. ${ }^{13}$

Once all known sources of thermal noise are taken into account, the integration time required to perform a UFF/WEP test to $10^{-17}$ with a signal to noise ratio of 2 is of about 3 hours. ${ }^{14}$ As a result, a reliable $10^{-17}$ test can be performed in 1 day (corresponding to 15 revolutions around the Earth and to 8 measurement cycles). During a 9-month mission, with the spacecraft in sun synchronous orbit and the spin axis fixed in space because of a very high rotation energy, classical systematic effects can be discriminated from a violation signal on the basis of their specific signature as determined by celestial mechanics. ${ }^{14}$

The "GG on Ground" (GGG) rotating experiment ${ }^{12}$ has reached, as a prototype of GG (i.e. after demodulation to $1.7 \cdot 10^{-4} \mathrm{~Hz}$ frequency of the target signal in space) a sensitivity of $8.9 \cdot 10^{-12}$.

A UFF/WEP test in space to $10^{-15}$ will soon be performed by the French $\mu \mathrm{SCOPE}$ mission $^{16}$ with contribution from ESA. The integration time required to reach this target is $1.4 \mathrm{~d}$; with the same level of thermal noise it would need a $10^{4}$ times longer integration time to reach $10^{-17}$, which is not feasible. ${ }^{15}$ As soon as $\mu \mathrm{SCOPE}$ data will be available the question as to "What next?" will arise.

\section{References}

1. A. M. Nobili, D. M. Lucchesi, M. T. Crosta, M. Shao, S. G. Turyshev, R. Peron, G. Catastini, A. Anselmi and G. Zavattini, Am. J. Phys. 81, 527 (2013).

2. A. Einstein, Annalen der Physik (Ser. 4) 49, 769-822 (1916); The foundation of the general theory of relativity in The Principle of Relativity (Dover Pub. Inc. N. Y., USA, 1952) p. 109.

3. T. Quinn, H. Parks, C. Speake and R. Davis1, Phys. Rev. Lett. 111, 101102 (2013).

4. P. G. Roll, R. Krotkov and R. H. Dicke, Ann. Phys. 26, $442-517$ (1964).

5. V. B. Braginsky and V. I. Panov, Sov. Phys. JEPT 34, 463-466 (1972).

6. S. Baeßler, B. R. Heckel, E. G. Adelberger, J. H. Gundlach, U. Schimidt and H. E. Swanson, Phys. Rev. Lett. 83, 3585 (1999).

7. S. Schlamminger, K.-Y. Choi, T. A. Wagner, J. H. Gundlach and E. G. Adelberger, Phys. Rev. Lett. 100, 041101 (2008).

8. T. D. Wagner, S. Shlamminger, J. Gundlach and E. G. Adelberger, Class. Quantum Grav. 29, 184002 (2012).

9. S. Carusotto, V. Cavasinni, A. Mordacci, F. Perrone, E. Polacco, E. Iacopini and G. Stefanini, Phys. Rev. Lett. 69, 1722 (1992).

10. V. Iafolla, E. C. Lorenzini, V. Milyukov and S. Nozzoli, Rev. Sci. Instrum. 69, 4146 (1998).

11. R. D. Reasenberg, B. R. Patla, J. D. Phillips and R. Thapa, Class. Quantum Grav. 29, 184013 (2012).

12. A. M. Nobili, M. Shao, R. Pegna, G. Zavattini, S. G. Turyshev, D. M. Lucchesi, A. De Michele, S. Doravari, G. L. Comandi, T. R. Saravanan, F. Palmonari, G. Catastini and A. Anselmi, Class. Quantum Grav. 29, 184011 (2012).

13. R. Pegna, A.M. Nobili, M. Shao, S.G. Turyshev, G. Catastini, A. Anselmi, R. Spero, S. Doravari, G. L. Comandi and A. De Michele, Phys. Rev. Lett. 107, 200801 (2011). 
14. A. M. Nobili, R. Pegna, M. Shao, S. G. Turyshev, G. Catastini, A. Anselmi, R. Spero, S. Doravari, G. L. Comandi, D. M. Lucchesi and A. De Michele, Phys. Rev. D 89, 042005 (2014).

15. A. M. Nobili et al., in preparation (2014).

16. P. Touboul, G. Metris, V. Lebat and A. Robert, Class. Quantum Grav. 29184010 (2012). 